\title{
NP-completeness Results for Partitioning a Graph into Total Dominating Sets
}

\section{Koivisto, Mikko}

Springer International Publishing AG

2017

Koivisto , M , Laakkonen , P \& Lauri , J 2017 , NP-completeness Results for Partitioning a Graph into Total Dominating Sets . in Y Cao \& J Chen (eds), Computing and Combinatorics : 23rd International Conference, COCOON 2017, Hong Kong, China, August 3-5, 2017, Proceedings . Lecture Notes in Computer Science, vol. 10392 , Springer International Publishing AG, Cham , pp. 333-345, International Computing and Combinatorics Conference, Hong Kong , China , 03/08/2017 . https://doi.org/10.1007/978-3-319-62389-4_28

http://hdl.handle.net/10138/307255

https://doi.org/10.1007/978-3-319-62389-4_28

acceptedVersion

Downloaded from Helda, University of Helsinki institutional repository.

This is an electronic reprint of the original article.

This reprint may differ from the original in pagination and typographic detail.

Please cite the original version. 


\title{
NP-completeness results for partitioning a graph into total dominating sets
}

\author{
Mikko Koivisto $^{1}$, Petteri Laakkonen ${ }^{2}$, and Juho Lauri ${ }^{2 \star}$ \\ 1 University of Helsinki, Finland \\ mikko.koivisto@helsinki.fi \\ 2 Tampere University of Technology, Finland \\ \{petteri.laakkonen, juho.lauri\}@tut.fi
}

\begin{abstract}
A total domatic k-partition of a graph is a partition of its vertex set into $k$ subsets such that each intersects the open neighborhood of each vertex. The maximum $k$ for which a total domatic $k$-partition exists is known as the total domatic number of a graph $G$, denoted by $d_{t}(G)$. We extend considerably the known hardness results by showing it is NP-complete to decide whether $d_{t}(G) \geq 3$ where $G$ is a bipartite planar graph of bounded maximum degree. Similarly, for every $k \geq 3$, it is NP-complete to decide whether $d_{t}(G) \geq k$, where $G$ is a split graph or $k$-regular. In particular, these results complement recent combinatorial results regarding $d_{t}(G)$ on some of these graph classes by showing that the known results are, in a sense, best possible. Finally, for general $n$ vertex graphs, we show the problem is solvable in $2^{n} n^{O(1)}$ time, and derive even faster algorithms for special graph classes.
\end{abstract}

\section{Introduction}

Domination is undoubtedly one of the most intensively studied concepts in graph theory. Besides being a fundamental graph property, domination also routinely appears in real-world applications related to data transfer (see e.g., $[9,31,16]$ ).

Let $G=(V, E)$ be a graph. A dominating set of $G$ is a set of vertices $S \subseteq V$ such that every vertex in $V$ either is in $S$ or is adjacent to a vertex in $S$. The domination number of a graph $G$, denoted by $\gamma(G)$, is the size of a minimum dominating set in $G$. A classical variant of domination is the concept of total domination, introduced by Cockayne et al. [7] in 1980. Here, a set of vertices $D \subseteq V$ is a total dominating set of $G$ if every vertex in $V$ is adjacent to some vertex in $D$. The total domination number of $G$, denoted by $\gamma_{t}(G)$, is the size of a minimum total dominating set in $G$. Note that every total dominating set is also a dominating set, but the converse is not true in general. Given the centrality of the topic, much is known about total domination in graphs. For instance, Bollobás and Cockayne [5] proved that $\gamma_{t}(G) \leq 2 \gamma(G)$. For other combinatorial results, we refer the reader to the books $[18,17]$ and the recent monograph [22]. From the viewpoint of complexity, the problem of deciding whether a graph $G$

\footnotetext{
* Current affiliation: Bell Labs, Ireland. E-mail: juho.lauri@nokia.com
} 


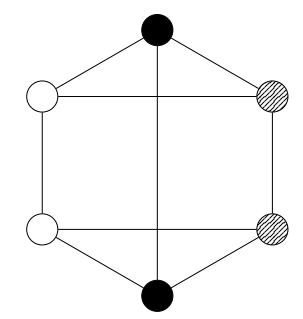

Fig. 1: The graph $G=\bar{C}_{6}$ has $d_{t}(G)=3$.

has a total dominating set of size at most $k$ is well-known to be NP-complete, even for bipartite graphs [27]. The reader interested in computational results is referred to the survey [20].

In this work, we focus on the problem of partitioning the vertex set of a graph into a maximum number of total dominating sets, a concept also introduced by Cockayne et al. [7]. For ease of presentation, we follow Zelinka [32] and use a definition rooted in graph colorings. A total domatic $k$-coloring is a partition of $V$ into $k$ color classes such that each vertex in $V$ is adjacent to a member from each class. The maximum $k$ for which a graph $G$ has a total domatic $k$ coloring is known as the total domatic number of $G$, and is denoted by $d_{t}(G)$. This is equivalent to the maximum number of total dominating sets the vertex set of $G$ can be partitioned into. For convenience, we can say a graph $G$ is total domatic $k$-colorable if $d_{t}(G) \geq k$. It is immediate from the definition that for any nontrivial graph $G$, we have that $1 \leq d_{t}(G) \leq \delta(G) \leq \Delta(G)$, where $\delta(G)$ and $\Delta(G)$ denote the minimum and maximum degree, respectively. For an illustration of the concept, consider the graph $G=\bar{C}_{6}$, i.e., the complement of a 6 -cycle (see Fig. 1). As $G$ is 3 -regular, we have that $d_{t}(G) \leq 3$. On the other hand, a total domatic 3-coloring of $G$ is straightforward to construct proving that $d_{t}(G)=3$.

Zelinka [33] proved that no minimum degree condition suffices to guarantee $d_{t}(G)=2$. Later on, Heggernes and Telle [19] showed that deciding whether $d_{t}(G) \geq 2$ is NP-complete for bipartite graphs. The study of total domatic number has regained attention lately. Recently, motivated by communication problems in large multi-robot networks, Chen et al. [6] reintroduced the concept under the name coupon coloring. In particular, they called the total domatic number the coupon coloring number. Subsequently, and seemingly unaware of the earlier work by Zelinka [32-34], the coupon coloring number was studied by Shi et al. [30]. In particular, Zelinka [32] determined the total domatic number of cactus graphs, majorizing Shi's et al. [30] result for cycles.

As further motivation, we mention a slight variation of an application in network science described by Abbas et al. [1]. Imagine a network of agents where each agent holds an instrument (e.g., thermometer, hygrometer, or so on). Moreover, interaction between agents is limited such that an agent can only communicate with its neighbors. As an agent requires access to each instrument, it needs to be copied across the agents to form a particular kind of dominating set. Indeed, to accommodate e.g., power limitations or system failures, we do 
not rely on the instrument an agent itself holds. Now, each instrument forms a total dominating set, and the maximum number of instruments that can be made available is precisely the total domatic number of the network. Abbas et al. [1] also stress that "... the underlying network topology of multirobot networks plays a crucial role in achieving the system level objectives within the network in a distributed manner." This motivates the study of the complexity of computing the total domatic number on restricted topologies. Furthermore, we also remark that Henning [20, Problem 12] calls for a deeper investigation of total domination on planar graphs.

Our results. We considerably extend the known hardness results for computing the total domatic number of a graph.

- For Section 3, our main result is that it is NP-complete to decide whether a bipartite planar graph $G$ of bounded maximum degree has $d_{t}(G) \geq 3$. This complements the recent combinatorial results of Goddard and Henning [14] who showed that no planar graph $G^{\prime}$ has $d_{t}\left(G^{\prime}\right)=5$. In other words, our result shows that it is unlikely one could have a polynomial-time characterization of planar graphs with described total domatic numbers.

- In Section 4, we prove that for every $k \geq 3$, it is NP-complete to decide whether a $k$-regular graph $G$ has $d_{t}(G) \geq 3$. In contrast, Akbari et al. [2] characterized the 3 -regular graphs $H$ with $d_{t}(H) \geq 2$. This is best possible in the sense that our hardness result gives strong evidence for the non-existence of a polynomial-time characterization of $k$-regular graphs with $d_{t}(G) \geq k$.

- In Section 5 we focus on chordal graphs. We begin by proving that it is NP-complete to decide whether $d_{t}(G) \geq 2$ where $G$ is a split graph. On a positive side, we show that the total domatic number can be computed in polynomial-time for threshold graphs.

- Finally, in Section 6, we give fast exact exponential-time algorithms for the problem. In particular, we show how the problem can be solved in $2^{n} n^{O(1)}$ time for general graphs, and derive even faster algorithms for special graph classes.

Statements whose proofs are located in the appendix are marked with $\star$.

\section{Preliminaries}

For a positive integer $n$, we write $[n]=\{1,2, \ldots, n\}$.

In what follows, we define the graph-theoretic concepts most central to our work. For graph-theoretic notation not defined here, we refer the reader to [10]. We also briefly introduce decision problems our hardness results depend on.

Graph parameters and classes. All graphs we consider are undirected and simple. For a graph $G$, we denote by $V(G)$ and $E(G)$ its vertex set and edge set, respectively. To reduce clutter, an edge $\{u, v\}$ is often denoted as $u v$. Two vertices $x$ and $y$ are adjacent (or neighbors) if $x y$ is an edge of $G$. The neighborhood of a vertex $v$, denoted by $N(v)$, is the set of all vertices adjacent to $v$. Let $G=(V, E)$. 
When we identify two distinct vertices $v, w \in V$, we obtain the graph with vertex set $V \backslash\{v, w\} \cup\{u\}$ and edge set $E \backslash\left\{\left\{v^{\prime}, v^{\prime \prime}\right\} \mid v^{\prime} \in V, v^{\prime \prime} \in\{v, w\}\right\} \cup\left\{\left\{v^{\prime}, u\right\} \mid\right.$ $v \notin\{v, w\}$ and $\left\{v^{\prime}, v\right\} \in E$ or $\left.\left\{v^{\prime}, w\right\} \in E\right\}$.

A vertex-coloring is a function $c: V \rightarrow[k]$ assigning a color from $[k]$ to each vertex of a graph $G=(V, E)$. The coloring is said to be proper if $c(u) \neq c(v)$ for every $u v \in E$. A graph $G$ is said to be $k$-colorable if there exists a proper vertexcoloring using $k$ colors for it. The minimum $k$ for which a graph $G$ is $k$-colorable is known as its chromatic number, denoted by $\chi(G)$. In particular, a 2-colorable graph is bipartite. Similarly, an edge-coloring is a function $f: E \rightarrow[k]$ assigning a color from $[k]$ to each edge. We say that $f$ is proper if two adjacent edges receive a distinct color under $f$. A graph $G$ is said to be $k$-edge-colorable if there exists a proper edge-coloring using $k$ colors for it. The minimum $k$ for which $G$ is $k$-edge-colorable is known as its chromatic index, denoted by $\chi^{\prime}(G)$.

A chord is an edge joining two non-consecutive vertices in a cycle. A graph is chordal if every cycle of length 4 or more has a chord. Equivalently, a graph is chordal if it contains no induced cycle of length 4 or more. A graph is a split graph if its vertex set can be partitioned into a clique and an independent set. It is known that all split graphs are chordal. A well-known subclass of split graphs is formed by threshold graphs, which are the graphs that can be formed from the empty graph by repeatedly adding either an isolated vertex or a dominating vertex (see [25, Theorem 1.2.4]).

Finally, we mention the following well-known structural measure for "treelikeness" of graphs. A tree decomposition of $G$ is a pair $\left(T,\left\{X_{i}: i \in I\right\}\right)$ where $X_{i} \subseteq V, i \in I$, and $T$ is a tree with elements of $I$ as nodes such that:

1. for each edge $u v \in E$, there is an $i \in I$ such that $\{u, v\} \subseteq X_{i}$, and

2. for each vertex $v \in V, T\left[\left\{i \in I \mid v \in X_{i}\right\}\right]$ is a tree with at least one node.

The width of a tree decomposition is $\max _{i \in I}\left|X_{i}\right|-1$. The treewidth of $G$, denoted by $\operatorname{tw}(G)$, is the minimum width taken over all tree decompositions of $G$.

Decision problems. Our main focus is the computational complexity of the following problem.

TOTAL Domatic $k$-PARTITION

Instance: A graph $G=(V, E)$.

Question: Can $V$ be partitioned into $k$ total dominating sets, i.e., is $d_{t}(G) \geq k$ ?

Our NP-completeness results are established by polynomial-time reductions from well-known coloring problems. We introduce them here for completeness. In both $k$-COLORING and Edge $k$-COLORING, the input is a graph $G=(V, E)$. In the former, we must decide whether $\chi(G) \leq k$, while in the latter, we are asked whether $\chi^{\prime}(G) \leq k$. Both problems are NP-complete for every $k \geq 3$ (see e.g., $[12,24])$. Finally, in the SET SplitTing problem, we are given a universe $U=[n]$ and a set system $\mathcal{F} \subseteq 2^{U}$. The task is to decide whether the elements of $U$ can be colored in 2 colors such that each member of $\mathcal{F}$ contains both colors. This problem is well-known to be NP-complete as well. 


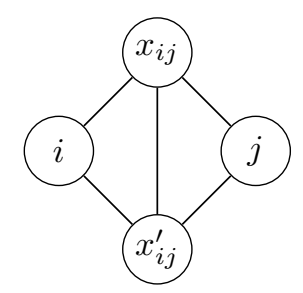

Fig. 2: The edge $i j$ replaced by a diamond in the construction of Lemma 2.

\section{Total domatic partitioning of planar graphs}

It is well-known that every planar graph $G$ has a vertex with degree at most five, so we have that $1 \leq d_{t}(G) \leq 5$. However, very recently, Goddard and Henning [14] proved that no planar graph $G$ has $d_{t}(G)=5$ by establishing the following tight bounds.

Theorem 1 (Goddard and Henning [14]). Any planar graph G has $1 \leq$ $d_{t}(G) \leq 4$. Moreover, these bounds are tight.

In the following, we prove that there is likely no straightforward (i.e., polynomialtime) characterization of planar graphs with specified total domatic numbers. More precisely, we show that it is NP-complete to decide whether a planar graph $G$ of bounded maximum degree has $d_{t}(G) \geq 3$. We first make the idea of the reduction clear, and then introduce slightly more complex gadgets that establish, using the same correctness argument, the same result for bipartite planar graphs of bounded maximum degree.

Lemma 2. 3-Coloring reduces in polynomial time to Total Domatic 3PARTITION.

Proof. Let $G$ be an instance of 3-Coloring. In polynomial time, we will create the following instance $G^{\prime}$ of Total Domatic 3-Partition, such that $G$ is 3 -colorable if and only if $G^{\prime}$ is total domatic 3-colorable.

The graph $G^{\prime}=\left(V^{\prime}, E^{\prime}\right)$ is obtained from $G$ by replacing each edge with a diamond (see Fig. 2), and by attaching to each vertex of $G$ a copy of $\bar{C}_{6}$ (see Fig. 1). Formally, we let $V^{\prime}=V \cup\left\{x_{i j}, x_{i j}^{\prime} \mid i j \in E\right\} \cup\left\{w_{v i} \mid v \in V, i \in[5]\right\}$. Set $E^{\prime}=D \cup C$, where

$$
\begin{aligned}
& D=\left\{i x_{i j}, i x_{i j}^{\prime}, x_{i j} x_{i j}^{\prime}, j x_{i j}, j x_{i j}^{\prime} \mid i j \in E\right\}, \\
& C=\left\{w_{v i} w_{v i+1}, w_{v 1} w_{v 5}, w_{v 2} w_{v 4}, v w_{v 1}, v w_{v 3}, v w_{v 5} \mid 1 \leq i<4, v \in V\right\} .
\end{aligned}
$$

This finishes the construction of $G^{\prime}$.

Let $c: V \rightarrow[3]$ be a proper vertex-coloring of $G$. We will construct a total domatic 3-coloring $c^{\prime}: V^{\prime} \rightarrow[3]$ as follows. We retain the coloring of the vertices in $V$, that is, $c^{\prime}(v)=c(v)$ for every $v \in V$. Then, as the degree of $x_{i j}$ is 3 , it holds that in any valid total domatic 3-coloring of $G^{\prime}$, the colors from [3] must be bijectively mapped to the neighborhood of $x_{i j}$; by symmetry, the same holds 


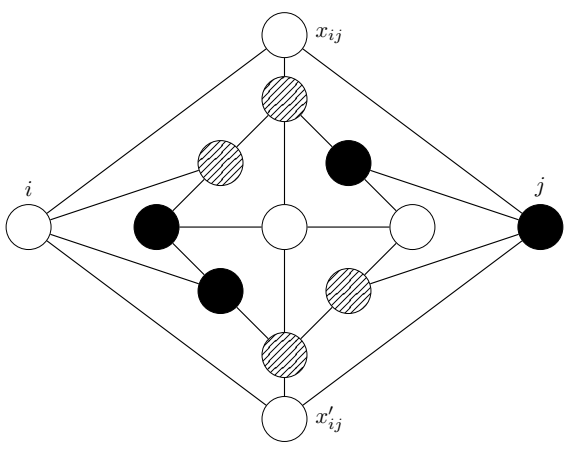

(a)

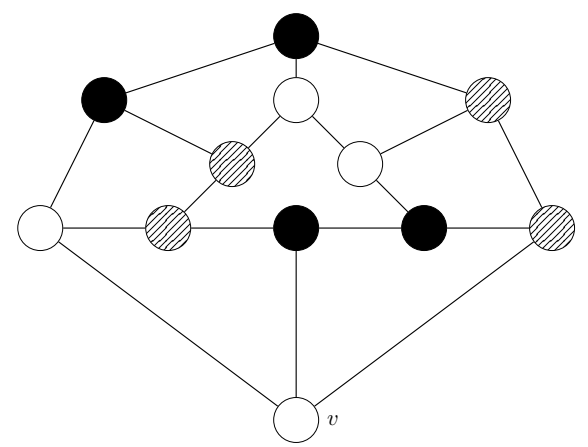

(b)

Fig. 3: (a) A replacment gadget for edge $i j$, and (b) a gadget each vertex $v$ is identified with. Both gadgets are planar and bipartite.

for $x_{i j}^{\prime}$. Then consider two adjacent vertices $i$ and $j$ in $G$. We set $c^{\prime}\left(x_{i j}\right)=$ $c^{\prime}\left(x_{i j}^{\prime}\right)=f$, where $f$ is the unique color in [3] neither $c(i)$ nor $c(j)$. Clearly, the neighborhood of both $x_{i j}$ and $x_{i j}^{\prime}$ contains every color from [3]. Finally, consider an arbitrary vertex $v \in V$. Without loss of generality, suppose $c(v)=1$. We will then finish the vertex-coloring $c^{\prime}$ as follows (see also Fig. 1):

$$
c^{\prime}\left(w_{v 5}\right)=1, c^{\prime}\left(w_{v 1}\right)=2, c^{\prime}\left(w_{v 3}\right)=2, c^{\prime}\left(w_{v 2}\right)=3, c^{\prime}\left(w_{v 4}\right)=3 .
$$

It is straightforward to verify that $c^{\prime}$ is indeed a total domatic 3-coloring of $G^{\prime}$.

For the other direction, suppose that there is a total domatic 3-coloring $c^{\prime}$ of $G^{\prime}$. Again, it holds that every color from [3] is bijectively mapped to the neighborhood of $x_{i j}$, for every $i$ and $j$. Moreover, it holds that $c^{\prime}\left(x_{i j}\right)=c^{\prime}\left(x_{i j}^{\prime}\right)$, implying that $c^{\prime}(i) \neq c^{\prime}(j)$. Thus, $c^{\prime}$ restricted to $G$ gives a proper 3 -coloring for $G$. This concludes the proof.

It is known that deciding whether a planar graph of maximum degree 4 can be properly 3-colored is NP-complete [12]. This combined with the previous lemma establishes the following.

Theorem 3. It is NP-complete to decide whether a planar graph $G$ of maximum degree 11 has $d_{t}(G) \geq 3$.

Corollary 4. It is NP-complete to decide whether a bipartite planar graph $G$ of maximum degree 19 has $d_{t}(G) \geq 3$.

Proof. To build $G^{\prime}$, we proceed with a construction similar to Lemma 2. However, for each edge $i j \in E(G)$, instead of a diamond, we construct the gadget shown in Fig. 3 (a). For each vertex $v \in V$, instead of $\bar{C}_{6}$, we identify $v$ with the gadget shown in Fig. 3 (b).

It is straightforward to verify that both gadgets are planar and bipartite. Clearly, $G^{\prime}$ is planar. Moreover, $G^{\prime}$ is bipartite as an odd cycle of $G$ has even length in $G$. Correctness follows by the same argument as in Lemma 2. 
We remark that Lemma 2 has consequences for the complexity of total domatic 3 -coloring graphs of bounded treewidth, and further consequences for planar graphs as well. Before proceeding, we briefly recall that a parameterized problem $I$ is a pair $(x, k)$, where $x$ is drawn from a fixed, finite alphabet and $k$ is an integer called the parameter. Then, a kernel for $(x, k)$ is a polynomial-time algorithm that returns an instance $\left(x^{\prime}, k^{\prime}\right)$ of $I$ such that $(x, k)$ is a YES-instance if and only if $\left(x^{\prime}, k^{\prime}\right)$ is a YES-instance, and $\left|x^{\prime}\right| \leq g(k)$, for some computable function $g: \mathbb{N} \rightarrow \mathbb{N}$. If $g(k)$ is a polynomial (exponential) function of $k$, we say that $I$ admits a polynomial (exponential) kernel (for more, see Cygan et al. [8]). We then recall the following earlier result.

Theorem 5 (van Rooij et al. [29]). For every $k \geq 1$, Total Domatic $k$-PARTITION parameterized by treewidth admits an exponential kernel.

In the following, we show that this is the best possible, i.e., there is no polynomial kernel under reasonable complexity-theoretic assumptions. We make the following observations regarding the gadget construction in Lemma 2.

Observation 6. It holds that $\mathrm{tw}\left(\bar{C}_{6}\right)=3$.

By identifying a $\bar{C}_{6}$ with a vertex of a bounded treewidth graph $G$, we do not considerably increase the treewidth of $G$.

Observation 7. Let $G$ be a graph of treewidth $k$, let $G^{\prime}$ be a graph of treewidth $k^{\prime}$, and let $H$ be the graph obtained through the identification of two vertices $v \in V(G)$ and $v^{\prime} \in V\left(G^{\prime}\right)$. Then $\operatorname{tw}(H) \leq \max \left\{k, k^{\prime}\right\}$.

Finally, Bodlaender et al. [4] proved that 3-COLORING does not admit a polynomial kernel parameterized by treewidth unless NP $\subseteq$ CoNP/poly. As the proof of Lemma 2 gives a parameter-preserving transformation guaranteeing $\operatorname{tw}\left(G^{\prime}\right) \leq$ $\operatorname{tw}(G)+3$, we have the following.

Theorem 8. Total Domatic 3-PARTition parameterized by treewidth does not admit a polynomial kernel unless $\mathrm{NP} \subseteq \mathrm{CONP} /$ poly.

Another consequence of Lemma 2 is captured by the following observation. For its statement, we recall the well-known exponential time hypothesis $(\mathrm{ETH})$, which is a conjecture stating that there is a constant $c>0$ such that 3-SAT cannot be solved in time $O\left(2^{c n}\right)$, where $n$ is the number of variables.

Corollary $9(\star)$. Total Domatic 3-PARTition for planar graphs cannot be solved in time $2^{o(\sqrt{n})}$ unless ETH fails, where $n$ is the number of vertices. However, the problem admits an algorithm running in time $2^{O(\sqrt{n})}$ for planar graphs.

\section{Total domatic partitioning of regular graphs}

Recently, Akbari et al. [2] characterized the 3-regular graphs with total domatic number at least two. In particular, they showed that these are precisely the 3regular graphs that do not contain a particular tree of maximum degree 3 as an 
induced subgraph. Moreover, it follows from the work of Henning and Yeo [21] that all $k$-regular graphs for $k \geq 4$ have $d_{t}(G) \geq 2$. We remark that the result of Akbari et al. [2] is the best possible in the sense that it is NP-complete to decide whether a $k$-regular graph $G$ has $d_{t}(G) \geq k$, for every $k \geq 3$. We establish this by reducing from EDGE $k$-COLORING on $k$-regular graphs, where $k \geq 3$, a problem shown to be NP-complete by Leven and Galil [24].

Essentially, our construction to follow is used already by Heggernes and Telle [19, Theorem 5], but we describe it in the appendix for completeness.

Theorem $10(\star)$. For every $k \geq 3$, it is NP-complete to decide whether a $k$ regular graph $G$ has $d_{t}(G) \geq k$.

\section{Total domatic partitioning of chordal graphs}

In the spirit of the previous sections, we begin by proving that total domatic coloring of chordal graphs is computationally difficult. More precisely, we show that for every $k \geq 2$, deciding whether $d_{t}(G) \geq k$ is NP-complete already for split graphs. The result is obtained by showing polynomial-time equivalence between this problem and HyPergraph RAInBOw $k$-COLORING, studied by Guruswami and Lee [15] among others. In the latter, we are given a universe $U=[n]$ and a set family $\mathcal{F} \subseteq 2^{U}$. The goal is to decide whether the elements of $U$ can be colored in $k$ colors such that each member of $\mathcal{F}$ contains each of the $k$ colors. The problem is equivalent to SET SPLiTting for $k=2$.

Theorem $11(\star)$. For every $k \geq 1$, Total Domatic $k$-Partition for split graphs is equivalent to HYPERGRAPH RAINBOW $k$-COLORING.

Theorem $12(\star)$. For every $k \geq 2$, it is NP-complete to decide whether a split graph $G$ has $d_{t}(G) \geq k$.

Given this negative result, it is interesting to consider split graphs with further restrictions on their structure. For instance, every complete graph is a split graph, and the total domatic number of complete graphs is known.

Proposition 13 (Shi et al. [30]). Let $G$ be the complete graph with $n$ vertices. Then $d_{t}(G)=\lfloor n / 2\rfloor$.

However, complete graphs have a very special structure: can we impose a weaker structural requirement on split graphs to obtain a graph class $G$ for which $d_{t}(G)$ can be computed in polynomial time? Before giving a positive answer to this question, we show the following.

Lemma 14. Let $G=(V, E)$ be a graph, and let $S$ be a subset of $V$ such that every $s \in S$ is a dominating vertex. Then $d_{t}(G) \geq \min \{\lfloor n / 2\rfloor,|S|\}$.

Proof. Denote $\ell=|S|$ and $h=|V \backslash S|$. Suppose first that $h \geq \ell$. We will prove that $d_{t}(G) \geq \ell$. Construct a total domatic $\ell$-coloring $c: V \rightarrow[\ell]$ as follows. Map

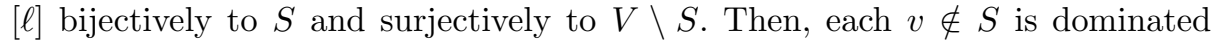


by each $s \in S$, and thus each color from [ $\ell]$ appears in the neighborhood of $v$. Similarly, by construction, [ $\ell]$ is mapped surjectively to $V \backslash S$ and $s \in S$ dominates each vertex not in $S$. Therefore, when $h \geq \ell$, we conclude that $d_{t}(G) \geq \ell$.

Finally, suppose that $h<\ell$. Let $q=\lfloor(\ell+h) / 2\rfloor=\lfloor n / 2\rfloor$, and choose $A \subseteq S$ such that $|A|=q$. Map $[\ell]$ bijectively to the vertices in $A$ and surjectively to $V \backslash A$. By the above argument, this is a valid total domatic $q$-coloring. Therefore, we have that $d_{t}(G) \geq q$, concluding the proof.

Theorem 15. Let $G=(V, E)$ be a connected threshold graph with $n$ vertices, and let $S$ be a subset of $V$ such that every $s \in S$ is a dominating vertex. Then $d_{t}(G)=\min \{\lfloor n / 2\rfloor,|S|\}$.

Proof. It is well-known that every threshold graph $G$ can be represented as a string $s(G)$ of characters $u$ and $j$, where $u$ denotes the addition of an isolated vertex, and $j$ the addition of a dominating vertex (see [25, Theorem 1.2.4]). Observe that if the last symbol of $s(G)$ is $u$, then $G$ is not connected and consequently $d_{t}(G)=0$. Thus, it holds that the last symbol of $s(G)$ is $j$.

Denote $\ell=|S|$ and $h=|V \backslash S|$. Suppose $h \geq \ell$, and consider the first occurrence of symbol $u$ in $s(G)$. The corresponding vertex has degree $\ell$, and no other vertex has degree less than $\ell$. Thus, $d_{t}(G) \leq \ell$. By Lemma 14, it follows that in this case, $d_{t}(G)=\ell$. Finally, suppose $h<\ell$. In the extremal case, $\ell=n$, i.e., each vertex of $G$ is a dominating vertex. By Proposition 13, we have that $d_{t}(G) \leq(\lfloor\ell+h\rfloor) / 2=\lfloor n / 2\rfloor$. On the other hand, Lemma 14 gives us a matching lower bound. This concludes the proof.

By the previous theorem, the total domatic number can be computed efficiently for threshold graphs.

Finally, despite Theorem 12, we observe that for some graphs $G$ we can always guarantee $d_{t}(G) \geq 2$.

Proposition 16. Let $G$ be an n-vertex Hamiltonian graph where $n$ is a multiple of four. Then $d_{t}(G) \geq 2$.

Proof. Let $v_{0} v_{1} \cdots v_{n}$ be a Hamiltonian cycle in $G$ such that $n \bmod 4=0$. Consider a vertex-coloring $c: V \rightarrow[2]$ such that $c\left(v_{i}\right)=c\left(v_{i+1}\right)=1$ and $c\left(v_{i+2}\right)=c\left(v_{i+3}\right)=2$ for $i=0,4, \ldots, n-4$. By construction, each neighborhood contains both colors 1 and 2 , so we are done.

\section{On exact algorithms for total domatic partitioning}

When a problem of interest is shown to be NP-complete, it motivates the consideration of alternative algorithmic approaches and easier special cases. Our results show that TOTAL Domatic $k$-PARTITION remains hard for several special cases. In addition, parameterization (see, e.g., [8]) by the number of colors $k$ seems uninteresting since the problem remains NP-complete for constant values of $k$. These observations further motivate the study of exact (exponential-time) algorithms. A brute-force algorithm tries every possible $k$-coloring and outputs 
YES if and only if one of the $k$-colorings is a total domatic $k$-coloring. Such an algorithm runs in time $k^{n} n^{O(1)}$, where $n$ is the number of vertices. Can we do considerably better? In what follows, we show this to be the case, and give even faster algorithms for special graph classes.

To obtain a moderately exponential algorithm, we apply a result of Björklund et al. [3] for the Set PARTition problem. In this problem we are given a universe $U=[n]$, a set family $\mathcal{F} \subseteq 2^{U}$, and an integer $k$. The task is to decide whether $U$ admits a partition into $k$ members of $\mathcal{F}$. Using algebraic methods, Björklund et al. showed the following.

Theorem 17 (Björklund et al. [3, Thms. 2 and 5]). Set Partition can be solved in $2^{n} n^{O(1)}$ time. If membership in $\mathcal{F}$ can be decided in $n^{O(1)}$ time, then SET PARTition can be solved in $3^{n} n^{O(1)}$ time and $n^{O(1)}$ space.

We apply this result to the set family consisting of all total dominating sets of a given graph. Since we can decide in polynomial time whether a given set of vertices is a total dominating set, we get the following.

Corollary 18. Total Domatic $k$-PARtition can be solved in $3^{n} n^{O(1)}$ time and polynomial space. In exponential space, the time can be improved to $2^{n} n^{O(1)}$.

We note that Björklund et al. give a similar application to domatic number. Relying on sophisticated algorithms for enumerating minimal dominating sets due to Fomin et al. [11], they further improve the polynomial-space results by lowering the constant of the exponential from 3 to 2.8718. Currently, the lowest constant is 2.7139, due to Nederlof et al. [26].

For total domatic number we discover another way to improve the polynomial-space algorithm, however, restricting ourselves to regular graphs. We get the following result by a simple reduction to graph coloring, for which the constant was recently improved to 2.2355 by Gaspers and Lee [13].

Theorem 19. One can decide in $O\left(2.2355^{n}\right)$ time and polynomial space whether a given $k$-regular graph $G$ with $n$ vertices has $d_{t}(G) \geq k$.

Proof. Define a graph $G^{\prime}=\left(V, E^{\prime}\right)$, where we put an edge between two vertices $u, v \in V$ exactly when they occur in the same neighborhood in $G$. It holds that $d_{t}(G)=k$ if and only if $\chi\left(G^{\prime}\right)=k$.

As noted by Chen et al. [6], Total Domatic $k$-Partition for $k=2$ corresponds to the well-known problem of hypergraph 2-coloring, also known as SET Splitting. To see this, we construct an instance of Set Splitting with the universe corresponding to the vertex set of the graph, and the set family to the neighborhoods. We obtain the following bound exploiting the algorithms of Nederlof et al. [26] for Set Splitting.

Theorem 20. One can decide in $O\left(1.8213^{n}\right)$ time and polynomial space whether a given graph $G$ with $n$ vertices has $d_{t}(G) \geq 2$. In exponential-space, the time can be improved to $O\left(1.7171^{n}\right)$. 


\section{Concluding remarks}

Our hardness results mirror those known for domatic number. Indeed, the computation of the domatic number was shown to be NP-complete for split graphs by Kaplan and Shamir [23], while hardness for bipartite planar graphs was proved by Poon et al. [28]. For positive results on special graph classes, results appear more scattered. For instance, it seems unknown whether domatic number can be solved in polynomial time for threshold graphs.

Concerning exact algorithms for total domatic number, an intriguing question is whether one can beat the $3^{n}$ time bound in polynomial space in general graphs. For domatic number the known algorithms achieve that via inclusion-exclusion and a branching algorithm that either lists minimal dominating sets or counts dominating sets. Currently we do not know whether these branching algorithms can be effectively adapted to total dominating sets.

Acknowledgments. This work was supported in part by the Academy of Finland, under Grant 276864 (M.K.), and by the Emil Aaltonen Foundation, under Grant 160138 N (J.L.).

\section{References}

1. Abbas, W., Egerstedt, M., Liu, C.H., Thomas, R., Whalen, P.: Deploying robots with two sensors in $K_{1,6}$-free graphs. Journal of Graph Theory 82(3), 236-252 (2016)

2. Akbari, S., Motiei, M., Mozaffari, S., Yazdanbod, S.: Cubic graphs with total domatic number at least two. arXiv preprint arXiv:1512.04748 (2015)

3. Björklund, A., Husfeldt, T., Koivisto, M.: Set partitioning via inclusion-exclusion. SIAM Journal on Computing 39(2), 546-563 (2009)

4. Bodlaender, H.L., Downey, R.G., Fellows, M.R., Hermelin, D.: On problems without polynomial kernels. Journal of Computer and System Sciences 75(8), 423-434 (2009)

5. Bollobás, B., Cockayne, E.J.: Graph-theoretic parameters concerning domination, independence, and irredundance. Journal of Graph Theory 3(3), 241-249 (1979)

6. Chen, B., Kim, J.H., Tait, M., Verstraete, J.: On coupon colorings of graphs. Discrete Applied Mathematics 193, 94-101 (2015)

7. Cockayne, E.J., Dawes, R.M., Hedetniemi, S.T.: Total domination in graphs. Networks 10(3), 211-219 (1980)

8. Cygan, M., Fomin, F.V., Kowalik, Ł., Lokshtanov, D., Marx, D., Pilipczuk, M., Pilipczuk, M., Saurabh, S.: Parameterized Algorithms. Springer (2015)

9. Dai, F., Wu, J.: An extended localized algorithm for connected dominating set formation in ad hoc wireless networks. IEEE Transactions on Parallel and Distributed Systems 15(10), 908-920 (2004)

10. Diestel, R.: Graph Theory. Springer-Verlag Heidelberg (2010)

11. Fomin, F.V., Grandoni, F., Pyatkin, A.V., Stepanov, A.A.: Combinatorial bounds via measure and conquer: bounding minimal dominating sets and applications. ACM Transactions on Algorithms 5(1), 9:1-9:17 (2008)

12. Garey, M., Johnson, D., Stockmeyer, L.: Some simplified NP-complete graph problems. Theoretical Computer Science 1(3), 237-267 (1976) 
13. Gaspers, S., Lee, E.: Faster graph coloring in polynomial space. ArXiv e-prints arXiv:1607.06201 (2016)

14. Goddard, W., Henning, M.A.: Thoroughly distributed colorings. arXiv preprint arXiv:1609.09684 (2016)

15. Guruswami, V., Lee, E.: Strong inapproximability results on balanced rainbowcolorable hypergraphs. In: Proceedings of the 26th Annual ACM-SIAM Symposium on Discrete Algorithms (SODA). pp. 822-836. SIAM (2015)

16. Han, B., Jia, W.: Clustering wireless ad hoc networks with weakly connected dominating set. Journal of Parallel and Distributed Computing 67(6), 727-737 (2007)

17. Haynes, T.W., Hedetniemi, S.T., Slater, P.J.: Domination in graphs: advanced topics. Marcel Dekker Inc., New York (1998)

18. Haynes, T.W., Hedetniemi, S.T., Slater, P.J.: Fundamentals of domination in graphs. CRC Press (1998)

19. Heggernes, P., Telle, J.A.: Partitioning graphs into generalized dominating sets. Nordic Journal of Computing 5(2), 128-142 (1998)

20. Henning, M.A.: A survey of selected recent results on total domination in graphs. Discrete Mathematics 309(1), 32-63 (2009)

21. Henning, M.A., Yeo, A.: 2-colorings in $k$-regular $k$-uniform hypergraphs. European Journal of Combinatorics 34(7), 1192-1202 (2013)

22. Henning, M.A., Yeo, A.: Total domination in graphs. Springer (2013)

23. Kaplan, H., Shamir, R.: The domatic number problem on some perfect graph families. Information Processing Letters 49(1), 51-56 (1994)

24. Leven, D., Galil, Z.: NP-completeness of finding the chromatic index of regular graphs. Journal of Algorithms 4(1), 35-44 (1983)

25. Mahadev, N.V.R., Peled, U.N.: Threshold graphs and related topics, vol. 56. Elsevier (1995)

26. Nederlof, J., van Rooij, J.M.M., van Dijk, T.C.: Inclusion/exclusion meets measure and conquer. Algorithmica 69(3), 685-740 (2014)

27. Pfaff, J., Laskar, R., Hedetniemi, S.T.: NP-completeness of total and connected domination and irredundance for bipartite graphs. Technical Report, Clemson University, Department of Mathematical Sciences 428 (1983)

28. Poon, S.H., Yen, W.C.K., Ung, C.T.: Domatic partition on several classes of graphs. In: Proceedings of the 6th International Conference on Combinatorial Optimization and Applications (COCOA). Lecture Notes in Computer Science, vol. 7402, pp. 245-256. Springer (2012)

29. van Rooij, J.M.M., Bodlaender, H.L., Rossmanith, P.: Dynamic programming on tree decompositions using generalised fast subset convolution. In: Proceedings of the 17th Annual European Symposium on Algorithms (ESA). Lecture Notes in Computer Science, vol. 5757, pp. 566-577. Springer (2009)

30. Shi, Y., Wei, M., Yue, J., Zhao, Y.: Coupon coloring of some special graphs. Journal of Combinatorial Optimization 33(1), 156-164 (2017)

31. Stojmenovic, I., Seddigh, M., Zunic, J.: Dominating sets and neighbor eliminationbased broadcasting algorithms in wireless networks. IEEE Transactions on Parallel and Distributed Systems 13(1), 14-25 (2002)

32. Zelinka, B.: Total domatic number of cacti. Mathematica Slovaca 38(3), 207-214 (1988)

33. Zelinka, B.: Total domatic number and degrees of vertices of a graph. Mathematica Slovaca 39(1), 7-11 (1989)

34. Zelinka, B.: Domination in generalized Petersen graphs. Czechoslovak Mathematical Journal 52(1), 11-16 (2002) 


\section{A Proofs for Section 3}

\section{Proof of Corollary 9}

Proof. Observe that the graph $G^{\prime}$ constructed in the proof of Lemma 2 has size linear in the size of the input graph $G$. The claimed lower bound then follows by a known chain of reductions originating from 3-SAT (see e.g., [8, Theorem 14.3]).

The claimed upper bound follows from combining the single-exponential dynamic programming algorithm on a tree decomposition of van Rooij et al. [29] with the fact that an $n$-vertex planar graph has treewidth $O(\sqrt{n})$.

\section{B Proofs for Section 4}

\section{Proof of Theorem 10}

Proof. We proceed with a polynomial-time reduction from EDGE $k$-COLORING on $k$-regular graphs, where $k \geq 3$. We will construct a $k$-regular graph $G^{\prime}$ such that $G$ is $k$-edge-colorable if and only if $d_{t}\left(G^{\prime}\right) \geq k$.

We replace every vertex of $G=(V, E)$ by a $k$-clique. More precisely, let $v \in V$ be a vertex with the neighbors $N(v)=\left\{v_{1}, v_{2}, \ldots, v_{k}\right\}$. Subdivide the edge $v v_{\ell}$ for $\ell \in[k]$ by a vertex $u_{\ell}$. Add all possible $\left(\begin{array}{c}k \\ 2\end{array}\right)$ edges between the vertices in $\left\{u_{1}, u_{2}, \ldots, u_{k}\right\}$ and delete $v$. This completes the construction of $G^{\prime}$. Clearly, as $G$ is $k$-regular, so is $G^{\prime}$.

Suppose $G$ is $k$-edge-colorable. A total domatic $k$-coloring $c^{\prime}: V\left(G^{\prime}\right) \rightarrow[k]$ for $G^{\prime}$ is constructed as follows. Suppose the edge $v v_{i}$ for some $i \in[k]$ was colored with color $f$ in $G$. We set $c^{\prime}\left(u_{i}\right)=f$. Now, since $G$ is $k$-edge-colorable, the vertex $u_{i}$ has $[k]$ mapped bijectively to its neighborhood, and we are done.

On the other hand, suppose $G^{\prime}$ is total domatic $k$-colored under $c^{\prime}$. Let $v \in V$, and denote by $C_{v}$ the $k$-clique formed by $u_{1}, \ldots, u_{k}$ in $G^{\prime}$. As the degree of $u \in C_{v}$ is $k$, it must be the case that $c^{\prime}$ assigns a distinct color to each $u$ in $C_{v}$, for otherwise there would be a vertex in $C_{v}$ whose neighborhood is missing at least one color in $[k]$. Now consider $w \in V$ adjacent to $v$ in $G$. Again, it must hold that $c^{\prime}$ assigns a distinct color to each $u^{\prime} \in C_{w}$. Moreover, it must be the case that $c^{\prime}(u)=c^{\prime}\left(u^{\prime}\right)$, for otherwise $N(u)$ would be missing at least one color in $[k]$. Thus, to construct an edge-coloring $c: E(G) \rightarrow[k]$, we let $c\left(v v_{i}\right)=c^{\prime}\left(u_{i}\right)$ for each $v \in V$ and $i \in[k]$. It follows that $c$ is a proper $k$-edge-coloring concluding the proof.

\section{Proofs for Section 5}

\section{Proof of Theorem 11}

Proof. The claimed equivalence is established by the following claims.

Claim 11.1. For every $k \geq 1$, Hypergraph Rainbow $k$-Coloring reduces in polynomial-time to Total Domatic $k$-PARTition for split graphs. 
Proof. Let $(U, \mathcal{F})$ be an instance of Hypergraph RAInbow $k$-COLORIng for any $k \geq 1$. We construct a split graph $G$ such that $U$ can be colored in $k$ colors such that each member of $\mathcal{F}$ contains each of the $k$ colors if and only if $d_{t}(G) \geq k$. Indeed, let $G=(C \cup I, E)$ where $C=\{x \mid x \in U\}, I=\{y \mid y \in \mathcal{F}\}$, and $E=\left\{x x^{\prime} \mid x, x^{\prime} \in C\right\} \cup\{y x \mid y \in I \wedge x$ is in the set $y$ corresponds to in $\mathcal{F}\}$. Clearly, it holds that $C$ forms a clique while $I$ is an independent set. Thus, $G$ is a split graph.

Suppose that each member of $\mathcal{F}$ contains each of the $k$ colors under a vertexcoloring $c: U \rightarrow[k]$. Then $c$ restricted to $C$ witnesses that $d_{t}(G) \geq k$. In the other direction, any total domatic $k$-coloring of $V(G)$ guarantees, in particular, that $[k]$ is mapped surjectively to $N(y)$ for each $y \in I$. This concludes the proof.

We then show the reverse direction.

Claim 11.2. For every $k \geq 1$, TOtal Domatic $k$-PARTition for split graphs reduces in polynomial-time to HYPERGRAPH RAINBOW $k$-COLORING.

Proof. Let $G=(C \cup I, E)$ be a split graph where $C$ induces a clique and $I$ forms an independent set. We construct an instance $(U, \mathcal{F})$ of HYPERGRAPH RAINBOW $k$-Coloring as follows. Let $U=V$, and for each $y \in I$ we add $N(y)$ to $\mathcal{F}$. We claim that $d_{t}(G) \geq k$ if and only if $U$ can be colored in $k$ colors such that each member of $\mathcal{F}$ contains each of the $k$ colors.

Observe that when $d_{t}(G) \geq k$ holds under some vertex-coloring $c$, it follows that each $y \in I$ has $[k]$ surjectively mapped to $N(y)$. Moreover, by the structure of $G$, each vertex of $N(y)$ is in $U$. Thus, $c$ restricted to $U$ guarantees that each member of $\mathcal{F}$ contains each of the $k$ colors. In the other direction, a rainbow $k$ coloring of $U$ witnesses that $d_{t}(G) \geq k$ since $[k]$ is surjectively mapped to $N(y)$ for each $y \in I$.

We have shown both directions, so the theorem follows.

\section{Proof of Theorem 12}

Proof. For $k=2$, the claim follows by observing that HyPERgRAPH RAInBow $k$-Coloring is equivalent to SET SPLitTing which is well-known to be NPcomplete.

For $k \geq 3$, we proceed with a polynomial-time reduction from EDGE $k$ COLORING on $k$-regular graphs. We construct a split graph $G^{\prime}$ such that the input graph $H$ is $k$-edge-colorable if and only if $d_{t}\left(G^{\prime}\right) \geq k$.

The split graph $G^{\prime}$ is constructed from the graph $H$ by subdividing every edge $e_{j} \in E(H)=\left\{e_{1}, e_{2}, \ldots, e_{m}\right\}$ with a new vertex $w_{j}$. These vertices are made adjacent by adding the edges $w_{i} w_{\ell}$, where $1 \leq i<\ell \leq m$. We can then verify that $H$ is a split graph with the set $W=V\left(G^{\prime}\right) \backslash V(H)$ forming a clique, and the set $I=V(H)$ being independent. We will then prove that $H$ is $k$-edge-colorable if and only if $d_{t}\left(G^{\prime}\right) \geq k$. 
Suppose $H$ is $k$-edge-colorable. Since $H$ is $k$-regular, a vertex $v \in I$ has degree exactly $k$. Moreover, $\delta\left(G^{\prime}\right)=k$, and thus $d_{t}(H) \leq k$. We will then show that $G^{\prime}$ is total domatic $k$-colorable. Indeed, we assign to the vertex $w \in W$ the color appearing on the edge corresponding to $w$ in $H$. Clearly, every color from $[k]$ appears in the neighborhood of $v \in I$. Finally, consider an arbitrary $w \in W$. By construction, it holds that $\operatorname{deg}(w)=m+1$. It follows, in particular, that $w$ is adjacent to each vertex in $N(u)$, where $u \in V(H)$. As $H$ is $k$-edge-colorable, we have that $[k]$ is mapped bijectively to $N(u)$, so $G^{\prime}$ is total domatic $k$-colorable.

For the other direction, again note that a vertex $v \in I$ has degree exactly $k$. Furthermore, as $G^{\prime}$ is total domatic $k$-colorable, there is a vertex-coloring under which every vertex adjacent to $v$ has received a distinct color. These adjacent vertices are all in $W$, and correspond to edges of $H$. Thus, we obtain a $k$-edgecoloring for $H$. This completes the proof. 